\title{
Ganciclovir-Resistant CMV Colitis in a Donor-Seronegative/ Recipient-Seronegative Liver Transplant Patient
}

\author{
Samuel O. Igbinedion, MD', Krunal Patel, $M D^{2}$, Hrishikesh Samant, MD², and \\ Gregory P. Wellman, MD³ \\ 'Department of Internal Medicine, Louisiana State University Health Sciences Center, Shreveport, LA \\ ${ }^{2}$ Department of Gastroenterology and Hepatology, Louisiana State University Health Sciences Center, Shreveport, LA \\ ${ }^{3}$ Delta Pathology, Shreveport, LA
}

\begin{abstract}
Cytomegalovirus (CMV) disease in liver transplant patients with donor-seronegative and recipient-seronegative (D-/R-) status is a rarity. Ganciclovir-resistant CMV disease in this population has not been reported in the literature. We present a 62-year-old man who underwent orthotopic liver transplant from a cadaveric donor. The patient's status was CMV D-/R-, and he had completed 90 days of valganciclovir therapy for CMV prophylaxis, yet he developed CMV disease. We highlight the danger of the liberal use of CMV prophylaxis in this subset of the liver transplant population. To our knowledge, this is the first report of ganciclovir-resistant (GanR) CMV disease in a liver transplant patient with CMV D-/R- status.
\end{abstract}

\section{INTRODUCTION}

Human cytomegalovirus (CMV) infection is usually asymptomatic in immunocompetent hosts, but it can cause severe disease in immunocompromised transplant patients and patients with acquired immunodeficiency syndrome (AIDS).' CMV infection has been associated with an increased predisposition to acute or chronic rejection, graft fibrosis, and shorter duration of graft survival. ${ }^{2}$ Ganciclovir and valganciclovir are primarily used as prophylaxis in the transplant setting. Some of the initial manifestations of CMV infection include interstitial pneumonia, hepatitis, Guillain-Barre syndrome, meningoencephalitis, and myocarditis.' It is very rare for a transplant patient with donor-seronegative and recipient-seronegative (CMV D-/R-) status to acquire CMV infection, let alone for someone in this population to acquire ganciclovir-resistant (GanR) CMV infection.

\section{CASE REPORT}

A 62-year-old man presented for liver transplant evaluation. His medical history was notable for liver cirrhosis secondary to chronic hepatitis $C$ with complications of slight ascites and grade-2 esophageal varices status post banding. The patient underwent an orthotopic liver transplant from a cadaveric donor, and he was given a donorseronegative and recipient-seronegative (CMV D-/R-) status. Immediate post-transplant immunosuppression was achieved with tacrolimus, mycophenolate, and prednisone therapy. Valganciclovir therapy for CMV prophylaxis was completed 90 days after transplant.

The patient presented 4 months after transplant with acute, non-bloody diarrhea. He reported a history of multiple watery stools every day in the past week. He had presented about 2 weeks after transplant with a similar complaint, and the mycophenolate dose was decreased at that time. On this recurrent presentation, he denied any history of fever or chills, abdominal pain, hematochezia. A digital rectal exam revealed non-thrombosed external

ACG Case Rep J 2018;5:e102. doi:10.14309/crj.2018.102. Published online: December 19, 2018.

Correspondence: Samuel O. Igbinedion MD, Louisiana State University Health Sciences Center Shreveport, 1501 Kings Highway, Shreveport, LA 71103 (sigbi1@lsuhsc.edu).

(c) (1) $\Theta$ Copyright: $\odot 2018$ Igbinedion et al. This work is licensed under a Creative Commons Attribution-NonCommercial-NoDerivatives 4.0 BY NG International License. To view a copy of this license, visit http://creativecommons.org/licenses/by-nc-nd/4.O. 


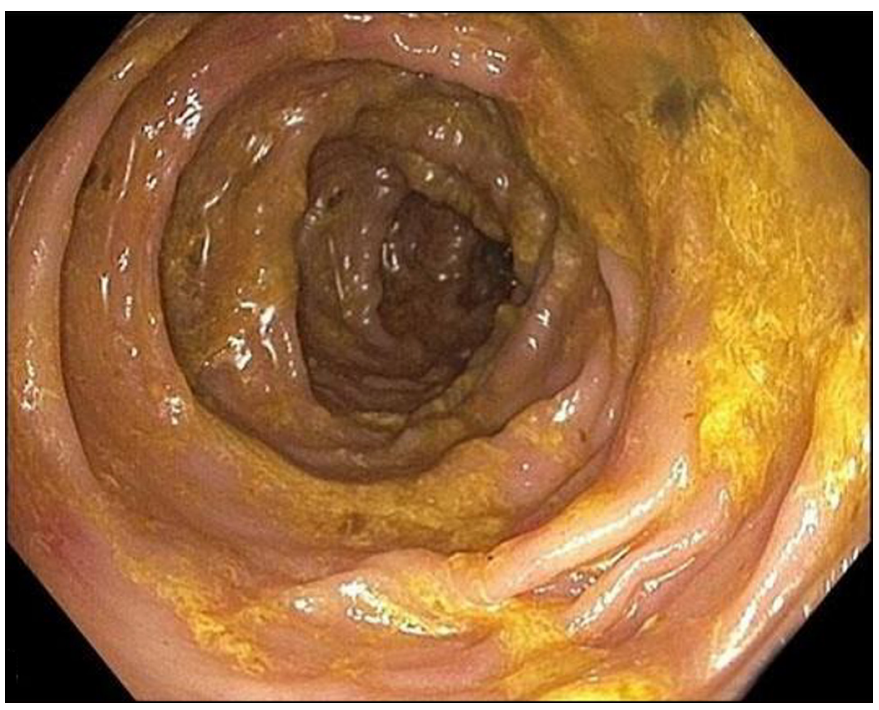

Figure 1. Flexible sigmoidoscopy revealing erythematous mucosa in the sigmoid colon and no evident ulcers. The sigmoid mucosal view is limited by poor preparation prior to the procedure.

hemorrhoids. Labs showed a white blood cell count $9.3 \times 10^{\%} /$ $\mathrm{L}$, baseline hemoglobin $11.1 \mathrm{~g} / \mathrm{dL}$, potassium $4.4 \mathrm{mmol} / \mathrm{L}$, and a significant rise in creatinine from 0.91 to $2.8 \mathrm{mg} / \mathrm{dL}$. Other significant labs were aspartate aminotransferase $311 \mathrm{U} / \mathrm{L}$, alanine aminotransferase $521 \mathrm{U} / \mathrm{L}$, alkaline phosphatase $290 \mathrm{U} / \mathrm{L}$, and total bilirubin $0.4 \mathrm{mg} / \mathrm{dL}$. The patient tested negative for Clostridum difficile infection. CMV/DNA quantitative pattern returned positive at $14,900 \mathrm{IU} / \mathrm{mL}$. Flexible sigmoidoscopy performed after appropriate fluid resuscitation showed erythematous mucosa in the sigmoid colon and no ulcers (Figure 1). Biopsies of the sigmoid colonic mucosa were obtained for histopathologic analysis. Results revealed a few fragments that demonstrated an increase in inflammatory cells within the lamina propria, minimal neutrophilic inflammation, apoptotic

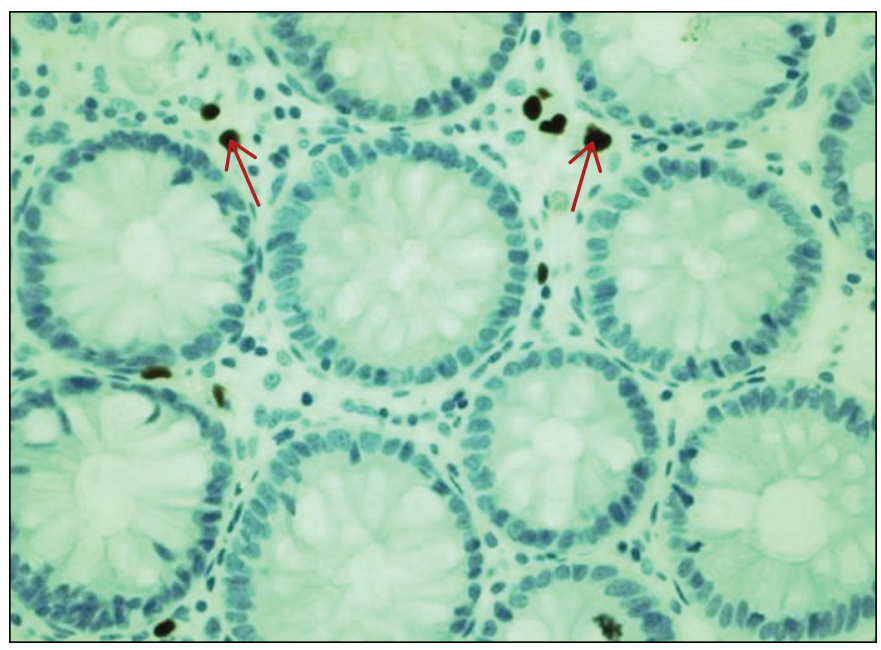

Figure 2. Immunohistochemical stain demonstrating positive cytomegalovirus immunoreactivity (arrows). debris, and occasional viral inclusions within endothelial and fibroblast cells in the lamina propria. Immunohistochemical stain for CMV returned positive (Figure 2). These findings correlated with a diagnosis of CMV colitis. Liver biopsy, performed because of elevated transaminases in the setting of CMV infection, revealed mild portal inflammation, which was deemed indeterminate for rejection. Immunohistochemical stain on the liver biopsy for CMV returned negative. The patient was treated with supportive care and also started on intravenous ganciclovir for 2 weeks followed by 4 weeks of valgancyclovir for a total of 6 weeks of therapy.

The patient was seen after initiation of therapy plan. Genotype studies showed mutations in CMV UL 97 and resistance to ganciclovir, indicative of infection with GanR CMV. The valganciclovir was discontinued at that time, and the patient was started on intravenous foscarnet. Repeat follow-up after 2 weeks returned a CMV PCR-negative status with normalization of liver enzymes. The patient reported resolution of diarrhea.

\section{DISCUSSION}

CMV is a pervasive double-stranded DNA virus that infects $60-100 \%$ of humans with primary CMV infection, commonly in the early years of life. ${ }^{3}$ It is the most common viral infection that affects liver transplant recipients. Liver transplant recipients with donor-seropositive and recipient-seronegative $(\mathrm{D}+/ \mathrm{R}-)$ status are at an increased risk of primary CMV infection. ${ }^{4}$ In the absence of antiviral prophylaxis, CMV infection in liver transplant recipients typically occurs during the first 3 months after the transplant. ${ }^{2}$ This can be tragic because it increases allograft rejection and thus the mortality rate in the transplant population., ${ }^{2,5}$ The incidence varies, depending on the CMV serologic status of the donor and recipient. The highest incidence is reported as $44-65 \%$ in the CMV D+/Rpopulation, and the lowest incidence reported is $1-2 \%$ in the CMV D-/R-population. ${ }^{4,6}$

The 1-2\% of the CMV D-/R- transplant population develops CMV disease either through natural transmission or blood transfusion. ${ }^{4}$ These patients are postulated to have the lowest risk of CMV infection, and routine use of prophylaxis is not generally recommended. ${ }^{4.7}$ However protocols for CMV prophylaxis, including the choice of drug and duration, varies across transplant centers. ${ }^{8}$ Our patient completed 3 months of oral valganciclovir dosed at $900 \mathrm{mg}$ once daily for CMV prophylaxis. He presented after completion of therapy with a clinical manifestation of CMV infection, indicated by evidence of CMV colitis in the setting of CMV viremia on the sigmoid colonic biopsy. The likelihood of this transplant patient with a CMV D-/R- status acquiring CMV disease after prophylaxis was considered to be very minimal. 
GanR CMV is an emerging entity now encountered in solidorgan transplant recipients, although less commonly in liver transplant recipients. ${ }^{9}$ Risk factors associated with the development of GanR CMV include a CMV D+/R-status, history of prolonged exposure to ganciclovir ( $>6$ weeks), potent immunosuppression (especially patients exposed to anti-thymocyte globulin or daclizumab), suboptimal ganciclovir levels, and high viral load., 90 The development of GanR CMV has been postulated to be a multi-hit disease model involving some or all of these factors. ${ }^{10}$ The extent of contribution of each of these factors is unknown. The continuous levels of CMV replication in the setting of suboptimal levels of the antiviral agent, especially in the absence of a CMV-specific immune function, can lead to development of resistance in the CMV D+/R- population." The mechanism of resistance in the D-/R- population is unclear. The only likely risk factor for the development of GanR CMV in the patient encountered in this case is the prolonged exposure to ganciclovir. This is because universal prophylaxis in this population is not generally recommended. Effective CMV prevention methods that minimize or avoid forms of ganciclovir exposure in this population would likely lead to lower rates of resistance. Therefore, to decrease the risk of GanR CMV, an approach in the CMV D-/R-population would be to withhold CMV prophylaxis unless otherwise indicated on a case-by-case basis. This is reflected in the updated international consensus guidelines on the management of CMV in solid-organ transplantation. ${ }^{7}$ We have thus used this case as a teaching point to review our practice with regard to CMV prophylaxis in this population.

Another plausible mechanism of resistance in this patient is the possibility of a false-negative donor graft. ${ }^{22} \mathrm{Nam}$ et al. highlights how testing in the "serologic window" timeframe prior to the development of antibodies to an infection could yield a false negative result. ${ }^{12}$ Donors in this time period could transmit infection to the recipients despite a negative serologic test. Our patient's donor could possibly have been infected with CMV previously without developing antibodies, thus being in this window. The prolonged exposure to ganciclovir in the post-transplant period could explain the subsequent development of GanR CMV disease.

Several cases of GanR CMV has been reported in the CMV $\mathrm{D}+/ \mathrm{R}$ - population, with a reported incidence of $0.4 \%$ in liver transplant recipients in a recent retrospective case-control study involving solid-organ recipients. ${ }^{10}$ To our knowledge, there had been no previous documentation of a case of GanR CMV in the CMV D-/R- liver transplant population. Ours is the first documented case of GanR CMV in this population. It is important to note that prophylaxis has been shown to be a more effective strategy than preemptive therapy in preventing CMV disease. , $^{813}$ This report, however, should alert gastroenterologists about the danger of the liberal use of CMV prophylaxis in the CMV D-/R- liver transplant population, with a need for evidence-based guidelines on prophylaxis based on other risk factors.

\section{DISCLOSURES}

Author contributions: SO Igbinedion, K. Patel, and H. Samat wrote and edited the manuscript. G. Wellman revised and approved the final version of the manuscript. SO Igbinedion is the article guarantor.

Financial disclosure: None to report.

Informed consent was obtained for this case report.

Received January 30, 2018; Accepted September 12, 2018

\section{REFERENCES}

1. Crumpacker CS. Cytomegalovirus (CMV). In: Bennett JE, Dolin R, and Blaser MJ, ed. Mandell, Douglas, and Bennett's Principles and Practice of Infectious Diseases. Philadelphia, PA: Elsevier Inc; 2017:1738-53.

2. Ljungman P, Griffiths P, Paya C. Definitions of cytomegalovirus infection and disease in transplant recipients. Clin Infect Dis. 2002;34(8):1094-97.

3. Razonable RR, Emery VC. Management of CMV infection and disease in transplant patients. Herpes. 2004;11(3):77-86

4. Yadav SK, Saigal S, Choudhary NS, Saha S, Kumar N, Soin AS Cytomegalovirus infection in liver transplant recipients: Current approach to diagnosis and management. J Clin Exp Hepatol. 2017;7 (2):144-51.

5. Fishman JA. Infection in solid-organ transplant recipients. N Engl J Med. 2007:357(25):2601-14.

6. Razonable RR, van Cruijsen H, Brown RA, et al. Dynamics of cytomegalovirus replication during preemptive therapy with oral ganciclovir. $J$ Infect Dis. 2003;187(11):1801-8.

7. Kotton CN, Kumar D, Caliendo AM, et al. The third international consensus guidelines on the management of cytomegalovirus in solid-organ transplantation. Transplantation. 2018;102(6):900-31.

8. Bodro M, Sabé N, Lladó L, et al. Prophylaxis versus preemptive therapy for cytomegalovirus disease in high-risk liver transplant recipients. Liver Transpl. 2012;18(9):1093-99.

9. Limaye AP. Ganciclovir-resistant cytomegalovirus in organ transplant recipients. Clin Infect Dis. 2002;35(7):866-72.

10. Fisher CE, Knudsen JL, Lease ED, et al. Risk factors and outcomes of ganciclovir-resistant cytomegalovirus infection in solid organ transplant recipients. Clin Infect Dis. 2017;65(1):57-63.

11. Paya CV. Prevention of cytomegalovirus disease in recipients of solidorgan transplants. Clin Infect Dis. 2001;32(4):596-603.

12. Nam H, Nilles KM, Levitsky J, Ison MG. Donor derived viral infections in liver transplantation. Transplantation. 2018;102(11):1824-36.

13. Witzke $O$, Nitschke M, Bartels $M$, et al. Valganciclovir prophylaxis versus preemptive therapy in cytomegalovirus-positive renal allograft recipients: Long-term results after 7 years of a randomized clinical trial. Transplantation. 2018;102(5):876-82. 